\title{
La contribution de Karl Löwith à l'anthropologie
}

\section{Guillaume Fagniez}

\section{OpenEdition}

\section{Journals}

Édition électronique

URL : http://journals.openedition.org/alter/377

DOI : 10.4000/alter.377

ISSN : 2558-7927

\section{Éditeur :}

Association ALTER, Archives Husserl (CNRS-UMR 8547)

\section{Édition imprimée}

Date de publication : 1 novembre 2015

Pagination : 98-115

ISBN : 978-2-9550449-1-9

ISSN : 1249-8947

Référence électronique

Guillaume Fagniez, «La contribution de Karl Löwith à l'anthropologie », Alter [En ligne], 23 | 2015, mis en ligne le 01 décembre 2017, consulté le 26 avril 2019. URL : http://journals.openedition.org/ alter/377 ; DOI : 10.4000/alter.377 


\section{LA CONTRIBUTION DE KARL LÖWITH À L'ANTHROPOLOGIE}

\section{Guillaume Fagniez}

L'œuvre de Karl Löwith est connue dans le monde francophone, à travers quelques grands textes, comme celle d'un interprète de Nietzsche (Nietzsche: Philosophie de l'éternel retour du même, 1935), d'un historien de la philosophie allemande du XIX siècle (De Hegel à Nietzsche, 1940), d'un disciple critique de Heidegger (Ma vie en Allemagne avant et après 1933, 1940), enfin d'un intervenant majeur dans le débat contemporain sur la sécularisation (Histoire et Salut, 1949). Ces quelques titres font deviner - au-delà de la silhouette du brillant polygraphe qu'ils esquissent - quelle confrontation avec les fondements de la modernité se joue dans l'ensemble des travaux de Löwith. Néanmoins, la relative dispersion des textes laisse ouverte la question de la cohérence profonde de son œuvre. À cet égard, l'hypothèse peut être formulée que l'unité de l'œuvre serait à chercher dans l'un de ses aspects qui a jusqu'ici peu retenu l'attention des lecteurs francophones, à savoir dans le projet explicitement anthropologique qui a été celui du philosophe depuis l'époque de sa thèse d'habilitation parue en 1928 sous le titre Das Individuum in der Rolle des Mitmenschen. Ein Beitrag zur anthropologischen Grundlegung der ethischen Probleme. Avec cet ouvrage, publié la même année que les deux grandes références de l'anthropologie philosophique du $\mathrm{XX}^{\mathrm{e}}$ siècle, Die Stellung des Menschen im Kosmos de Max Scheler et Die Stufen des Organischen und der Mensch de Helmut Plessner, Löwith paraît à première vue épouser le «tournant anthropologique » intervenu dans la pensée allemande dans les années vingt ${ }^{1}$. En se soustrayant aux cadres problématiques de la phénoménologie à l'école de

${ }^{1}$ Cf. J. Fischer, Philosophische Anthropologie. Eine Denkrichtung des 20. Jahrhunderts, Fribourg/ Munich, Karl Alber, 2008, p. 94. 
laquelle il s'est formé, et en brisant ce qu'on a pu appeler son « interdit anthropologique », Löwith se saisit à son tour de la question "Qu'estce que l'homme? » Toutefois, sa manière d'interroger la nature de l'homme contient une position originale, notamment en ce qu'elle tend à accorder, à la faveur d'un renversement du questionnement, la place centrale à la nature plutôt qu'à l'homme lui-même. Dans la chronologie de l'œuvre de Löwith en effet, deux temps se succèdent, qui correspondent aux deux moments de $\mathrm{l}^{\prime}$ « anthropologie philosophique » repérés par Odo Marquard dans son histoire du concept : d'abord, un premier «tournant vers le monde de la vie » suscitant l'adoption d'un " point de vue anthropologique»; puis une vigoureuse « récusation de la philosophie de l'histoire $»^{2}$ qui engage en même temps une révision complète de la conception du rapport entre l'homme et la nature. Ces deux moments successifs sont chez Löwith séparés par un renversement qui, plaçant la nature au centre de l'interrogation anthropologique, rejette à la périphérie l'être même en question : l'homme. C'est au point de pivotement de ce tournant, où semble se concentrer son sens, que nous chercherons à caractériser à grands traits l'originalité de la contribution de Karl Löwith à l'anthropologie.

\section{Le point de vue anthropologique}

Il convient tout d'abord de relativiser la concomitance de la tournure anthropologique prise par les recherches de Löwith et de l'émergence de l'anthropologie philosophique chez Scheler et Plessner: l'histoire de cette dernière ne permet guère de mettre en évidence une véritable communauté de pensée fondée sur des jeux d'influences réciproques. À cette époque, ces deux références semblent ne jouer qu'un rôle mineur dans la pensée de Löwith: le travail de synthèse consacré par ce dernier à Scheler paraît en $1935^{3}$, et c'est seulement dans les années cinquante qu'il se lie plus étroitement avec Plessner, comme lui «rémigrant» en Allemagne. Déterminante en revanche est la réaction à l'enseignement de Heidegger, auquel Löwith a assisté à partir de 1919, à Fribourg puis à Marbourg. Ainsi, lorsque Löwith présente sa

\footnotetext{
${ }^{2}$ Cf. O. Marquard, «Sur l'histoire du concept philosophique d"“anthropologie" depuis la fin du XVIII ${ }^{\mathrm{e}}$ siècle", in Des difficultés avec la philosophie de l'histoire, trad. O. Mannoni, Paris, Maison des sciences de l'homme, 2002, p. 137-169, ainsi que l'article "Anthropologie » du même auteur dans Historisches Wörterbuch der Philosophie, J. Ritter éd., Darmstadt, Wissentschaftliche Buchgesellschaft, 1971, vol. 1, col. 362-374.

${ }^{3}$ Cf. K. Löwith, Max Scheler und das Problem einer philosophischen Anthropologie, in Mensch und Menschenwelt. Beiträge zur Anthropologie, Sämtliche Schriften Band 1 (cité dans ce qui suit: SS 1), K. Stichweh éd., Stuttgart, J. B. Metzler, 1981, p. 219-242.
} 
thèse d'habilitation et annonce que la «fondation des problèmes éthiques » qui y est tentée «sera désignée comme une fondation anthropologique », c'est, précise-t-il, « afin de la démarquer [du] concept purement ontologique de la phénoménologie $»^{4}$. Ces analyses, anthropologiques «dans leur méthode et dans leur contenu ${ }^{5}$, sont placées sous la tutelle de Feuerbach, figure par excellence de $l^{\prime}$ «humanisation $»^{6}$ de la philosophie, et sous le signe de son « principe »: mettre en œuvre la philosophie «du point de vue de l'anthropologie »? Ce principe, selon la lecture de Löwith, revêt une double signification : la reconduction, en dernière instance, de la pensée abstraite à l'intuition sensible («sensualisme»); l'implication, en toute pensée, d'un autre homme que le Moi pensant, d'un «Tu» par lequel seulement il y a «Je» («altruisme»). À l'évidence, dans sa thèse, Löwith s'inscrit en faux contre ce qui lui paraît être chez Heidegger un "solipsisme existential », et le rapport de l'homme à son autre - au Mitmensch - est une dimension essentielle du projet anthropologique comme tel. Néanmoins, lorsque la méthode est explicitée, c'est le rapport à la «sensibilité » qui est accentué : l'enquête se reconnaît ainsi comme « source » l'expérience de «l'homme » en ce qu'elle a d'immédiatement accessible. En ce sens, elle est descriptive, en l'occurrence de «l'attitude humaine fondamentale» et des structures de l'expérience humaine en tant $q^{\prime}$ 'elle est fondamentalement "en rapport à » autrui ${ }^{8}$. Les analyses du Miteinandersein sont également anthropologiques au sens davantage thématique où elles entendent partir de «ce qui dans les phénomènes quotidiens de la vie humaine demande de fait à être interrogé »: «elles reviennent, afin de poser des bases sur un plan anthropologique, aux configurations structurales les plus élémentaires de la vie humaine - à des trivialités $»^{9}$.

La principale visée de cette recherche anthropologique est ainsi l'inscription de la pensée en une dimension d'immanence caractéristique de la vie humaine. C'est pourquoi la réaction à l'enseignement

\footnotetext{
${ }^{4} \mathrm{~K}$. Löwith, Das Individuum in der Rolle des Mitmenschen, SS 1, p. 11. Heidegger lui-même, directeur de la thèse, avait recommandé une "distinction plus claire entre les conceptions "ontologique" et "anthropologique" de l'existence humaine » (SS 1, p. 473). Le sous-titre de la première édition évoquant une "fondation anthropologique des problèmes éthiques " - et non plus "phénoménologique » comme c'était le cas dans la version de la thèse déposée fin 1927 - a lui aussi été suggéré par Heidegger, mais moins aux fins d'une telle clarification que dans le souci de la poursuite de la carrière académique de Löwith à Marbourg (cf. SS 1, p. 469 sq.).

${ }^{5} \mathrm{~K}$. Löwith, Das Individuum in der Rolle des Mitmenschen, SS 1, p. 12.

${ }^{6}$ L. Feuerbach, Principes de la philosophie de l'avenir, in Manifestes philosophiques, trad. L. Althusser, Paris, PUF, 1960, p. 128.

${ }^{7}$ K. Löwith, Das Individuum in der Rolle des Mitmenschen, SS 1, p. 21.

${ }^{8}$ Ibid., p. 13.

${ }^{9}$ Idem.
} 
de Heidegger est profondément ambivalente, à l'aune d'une transformation dont Löwith a lui-même été le témoin. La pensée du jeune Heidegger se présente en effet d'abord comme une phénoménologie de la vie : l'unique objet de la philosophie, pour Heidegger, est la «vie» humaine en sa "factualité » caractéristique. Or, si une telle question paraît rétrospectivement devoir se déployer comme question du sens même de $l^{\prime}$ " être », la phénoménologie, irréductible à une préhistoire de la question de l'être, peut néanmoins être entendue comme une interrogation sur l'essence de l'homme. Elle se présente en effet initialement comme une description de la vie humaine indépendante des transgressions métaphysiques de l'expérience et rétive aux réductions propres aux sciences positives; plus radicalement, elle s'oppose aux dénaturations que font subir à la vie la philosophie théorique depuis Platon et la philosophie de la conscience de Descartes à Husserl. Parmi les "recouvrements" ayant contribué à occulter le «phénomène primordial » de la vie, «l'idée d'homme » joue assurément un rôle majeur: Heidegger, dès 1923, renvoie l'anthropologie de Scheler à la généalogie théologico-philosophique de son concept d'humanité, dont elle reconduit la bipartition fondamentale de l'animal rationale ${ }^{10}$. Toutefois, $\mathrm{c}^{\prime}$ est bien le même phénomène qui, occulté par l'anthropologie, est visé par la phénoménologie, à telle enseigne que celle-ci peut se déployer au fil d'une "dé-struction » de celle-là, soit d'un démantèlement généalogique de l'anthropologie jusqu'à sa source: la vie en sa Faktizität, c'est-à-dire également en un monde. C'est en ce point que le projet anthropologique de Löwith peut s'amorcer et prendre son essor, et la description de la Mitwelt à laquelle procède la thèse de 1928 prend implicitement comme étalon celle de l'Umwelt opérée par Heidegger dès ses tout premiers cours ${ }^{11}$.

Löwith défend ainsi le «point de vue anthropologique» avec Heidegger, c'est-à-dire à partir de la focalisation sur la vie et à travers l'analyse des structures du "monde de la vie»; mais également contre Heidegger, en contestant l'orientation ontologique de la phénoménologie de la vie ${ }^{12}$. Deux principes de $1^{\prime}$ «herméneutique de la

\footnotetext{
${ }^{10}$ Cf. M. Heidegger, Ontologie. Hermeneutik der Faktizität, GA 63, p. 21-29; Ontologie. Herméneutique de la factivité, trad. A. Boutot, Paris, Gallimard, 2012, p. 42-52.

${ }_{11}$ Cf. en particulier M. Heidegger, Zur Bestimmung der Philosophie, GA 56/57, p. 70-73 et 84-94.

${ }^{12}$ Dans une lettre d'août 1927, Löwith présente implicitement son projet anthropologique comme plus fidèle à l'herméneutique de la Faktizität que l'analytique existentiale ne le serait ellemême. Cf. E. Donaggio, "Zwischen Nietzsche und Heidegger. Karl Löwiths anthropologische Philosophie des faktischen Lebens », Deutsche Zeitschrift für Philosophie, n 48, Berlin, De Gruyter, 2000/1, p. 48.
} 
Faktizität» sont ici adoptés, que Löwith entend mettre rigoureusement en œuvre : d'une part, la philosophie a vocation à interpréter la vie «à partir d'elle-même », sans emprunt à des schémas qui lui sont étrangers ; d'autre part, toute interprétation se déploie à partir d'un horizon qui demande à être explicité. Au nom de cette auto-interprétation conséquente de la vie, Löwith retourne contre Heidegger sa critique de la tradition philosophique. C'est qu'aux yeux de Löwith, la phénoménologie, en se tournant vers l'être - soit " rien d'étant » vise une instance parfaitement hétérogène à la vie, et partant renonce à elle. Une telle focalisation de la phénoménologie sur l'être a ainsi partie liée avec une détermination de l'homme qui l'arrache au faktisches Leben d'abord envisagé par Heidegger. Le primat est ici accordé à l'existential sur l'existentiel, à l'ontologique sur l'ontique, et cela en un double sens. D'une part, l'homme est conçu prioritairement à titre de possibilité d'être, plus exactement d'être soi-même, " en propre ». Est ici présupposé, selon Löwith, un «concept tout à fait déterminé de la nature de la vie humaine, à savoir celui selon lequel la "substance" de l'homme est son "existence", et la "consistance" (Bestand) de la vie humaine "l'autonomie (Selbstständigkeit) du soi existant" ${ }^{13}$. Si bien qu'il est dès lors possible d'abstraire l'existence « de » l'homme, et de se concentrer sur le Dasein « en » l'homme au titre d'une possibilité distincte de cette «humanité » ${ }^{14}$. D'autre part, le primat de l'ontologique est un primat de l'onto-logique: la Faktizität de la vie se voit entièrement annexée au sens, dès lors que l'existence se déploie à la mesure de son intelligence de l'être et de l'explicitation de cette dernière forgée au creuset d'une possibilité propre. L' « idéal d'existence » qui commande l'interprétation heideggérienne de la vie humaine correspond à une expérience bien déterminée, celle de «l'homme de science». Cette dernière constitue «le présupposé anthropologique de l'ontologie» par lequel s'inverse l'ordre naturel des priorités : aux yeux de Löwith, on ne peut envisager la vie humaine à la lumière de la conscience philosophante et dire avec Heidegger qu' «être homme c'est philosopher»-c'est-àdire «transcender $»^{15}$. Toute la réflexion doit au contraire s'élever sur la base de «l'être-homme antérieur et universel» dont l'une des

\footnotetext{
${ }^{13}$ K. Löwith, Phänomenologische Ontologie und protestantische Theologie, in Wissen, Glaube und Skepsis. Zur Kritik von Religion und Theologie, Sämtliche Schriften Band 3, Stuttgart, J. B. Metzler, 1985, p. 10.

${ }^{14}$ Cf. K. Löwith, Phänomenologische Ontologie und protestantische Theologie, SS 3, p. 17, et Die Einheit und die Verschiedenheit des Menschen, SS 1, p. 252-253.

${ }^{15}$ Tel est notamment le point de départ du cours prononcé par Heidegger au semestre d'hiver 1928-1929 Einleitung in die Philosophie, GA 27.
} 
multiples figures est la vie consacrée à la connaissance ${ }^{16}$. Ainsi, du point de vue de Löwith, les concepts élaborés par l'analytique existentiale procèdent de ces "déthéologisations formalisantes » mêmes que Heidegger reproche à Scheler ${ }^{17}$ : la transcendance du Dasein se laisse énoncer en un vocabulaire dont Löwith ne se prive pas de noter les résonances religieuses. Et si la théologie s'approprie sans difficultés l'analytique existentiale, ce n'est pas que cette dernière procure à la pensée des instruments conceptuels formels et neutres puisés sans préventions à même l'expérience, et partant d'application universelle, mais au contraire qu'elle repose sur une Aufhebung de la théologie qui s'est entièrement assimilé ce dont elle est la négation ${ }^{18}$.

Dans son texte de 1969 adressé à Heidegger pour ses quatre-vingts ans, Löwith revient une fois encore sur les motifs qui ont décidé l'adoption d'un point de vue anthropologique contre la problématique strictement ontologique. Après avoir rappelé ses études de biologie et sa sensibilité pour la « merveille d'organisation » que donne à voir un être vivant, il ajoute :

ce que je regrettais, dans la problématique ontologico-existentiale, c'était l'absence de la nature - autour de nous et en nous-mêmes. Mais lorsque la nature est absente, ce n'est pas un étant ou un domaine d'être parmi d'autres qui fait défaut, bien au contraire: l'entièreté de l'étant en tant que tel est manquée, et elle ne se laisse nullement ajouter après-coup à titre de complément. Car qu'est-ce que la nature si ce n'est une nature de tout étant dont la force créatrice fait advenir puis de nouveau disparaître tout ce qui est, y compris l'homme ${ }^{19}$ ?

Dans son livre de 1953 Heidegger, Denker in dürftiger Zeit, Löwith développe les différentes phases de ce qui constitue à ses yeux une indifférence caractéristique de la pensée heideggérienne à l'égard de la nature ${ }^{20}$. Mais dès la fin des années vingt sont nouées chez Löwith, dans son opposition à Heidegger, la nécessité d'une philosophie comme anthropologie et la référence à la nature ${ }^{21}$. Ce qui l'atteste remarquablement est le fait que Löwith aperçoit dans la détermi-

\footnotetext{
${ }^{16}$ K. Löwith, Phänomenologische Ontologie und protestantische Theologie, SS 3, p. 17.

${ }^{17}$ Cf. M. Heidegger, Ontologie. Hermeneutik der Faktizität, GA 63, p. 26 ; trad. citée p. 49.

${ }^{18}$ Cf. K. Löwith, Phänomenologische Ontologie und protestantische Theologie, SS 3, p. 2 sqq.

${ }^{19}$ K. Löwith, Die Natur des Menschen und die Welt der Natur, in Die Frage Martin Heideggers. Beiträge zu einem Kolloquium mit Heidegger aus Anlaß seines 80. Geburtstages, H.-G. Gadamer éd., Heidelberg, Sitzungsberichte der Heidelberger Akademie der Wissenschaften, 1969, p. 39-40.

${ }^{20}$ Cf. K. Löwith, Heidegger - Denker in dürftiger Zeit. Zur Stellung der Philosophie im 20. Jahrhundert, Sämtliche Schriften Band 8, Stuttgart, J. B. Metzler, p. 182-187.

${ }^{21}$ En témoigne notamment la lettre de Heidegger datée du 20 août 1927 citée par Löwith dans son texte de 1969 ; cf. K. Löwith, Die Natur des Menschen und die Welt der Natur, op. cit., p. 40 et M. Heidegger, «Trois lettres à Karl Löwith », Archives de philosophie, 73, 2010, p. 329.
} 
nation traditionnelle de l'homme comme animal rationale une vérité profonde qu'il importe de préserver et redécouvrir. Elle a en effet ceci de précieux qu'elle souligne l'« équivocité (Zweideutigkeit) ontologique de la vie humaine ${ }^{22}$. Cette dernière s'explicite dans les mêmes termes de la thèse de 1928 jusqu'à l'hommage à Heidegger de 1969, qui souligne à son adresse :

l'homme en tant que nature pensante ne peut vivre corporellement que dans la mesure où il prend lui-même en charge son existence; mais s'il peut luimême se prendre en charge, c'est que, par nature (von Natur aus), il est déjà là, il vit, se rapporte à lui-même et pense. La détermination traditionnelle de l'homme comme animal rationale que vous [Heidegger] avez combattue sur toute la ligne et reconduite à l'oubli de l'être lié à la métaphysique, fait ainsi retour; elle a l'avantage de ne pas déterminer l'homme de façon univoque, unitaire et unilatérale par l'âme et l'esprit, ou la conscience et l'existence, ou encore en tant que «là » de l'être, mais au contraire comme une dualité incarnée d'animalité et de rationalitée 23 .

La thèse de la «dualité » de l'essence de l'homme signifie avant tout une contestation de la détermination exclusive de l'homme à partir de sa dimension «logique », voire du simple primat de la conscience. À plusieurs reprises, Löwith se réfère à l'ouvrage de Karl Rosenkranz intitulé Psychologie oder Wissenschaft vom subjektiven Geist (1837) qui entend développer la «doctrine de l'esprit subjectif » de Hegel, lequel n'en avait donné qu'un exposé bref et dense au sein de son Encyclopédie des sciences philosophiques. Ce qui retient l'attention de Löwith, c'est l'analyse du sommeil qui s'y trouve élaborée. D'un côté, il faut accorder à Rosenkranz un primat méthodologique de la conscience, et c'est bien toujours un être éveillé qui peut s'interroger sur ses états, de veille comme de sommeil. Néanmoins, il convient également d'abonder en son sens lorsqu'il remarque qu'inconscience et sommeil caractérisent "l'état littéralement originel de l'homme avant la naissance », «ce premier éveil élémentaire ${ }^{24}$, et plus encore de contester la domination de «l'ontologie de la conscience ».

Le présupposé de l'ontologie de la conscience, de Descartes à Hegel et audelà, jusqu'à la philosophie de l'existence, selon lequel l'être conscient de lui-même et se rapportant à lui-même est également déterminant pour la compréhension de l'être inconscient ou purement et simplement vivant,

\footnotetext{
${ }^{22}$ K. Löwith, Das Individuum in der Rolle des Mitmenschen, SS 1, p. 33.

${ }^{23}$ K. Löwith, Die Natur des Menschen und die Welt der Natur, op. cit., p. 40-41.

${ }^{24}$ Cf. K. Löwith, Zur Frage einer philosophischen Anthropologie, SS 1, p. 340 sq., Die Natur des Menschen und die Welt der Natur (op. cit., p. 45-46) et Die Ausführung von Hegels Lehre vom Subjektiven Geist durch Karl Rosenkranz (Hegels philosophische Psychologie, D. Henrich éd., HegelStudien, Beiheft 19, 1979, p. 227-234.)
} 
un tel présupposé traditionnel n'est qu'une demi-vérité. Si l'on prend cette dernière pour la vérité tout entière, elle est alors fausse, car il y a un mode de l'existence humaine indépendant de la conscience éveillée et antérieur à l'être conscient de lui-même. Le fait que la conscience soit déterminante pour la compréhension de l'inconscient ne signifie pas qu'elle le soit également pour ce qu'est le vivant lui-même, soit autre chose et davantage qu'un simple manque de conscience. À supposer même que tout être préconscient, subconscient et inconscient soit disposé au devenir-conscient, l'autre moitié de la vérité complète n'en resterait pas moins vraie, à savoir que pendant notre vie et notre existence éveillées et conscientes, ce qui nous arrive a lieu la plupart du temps sans conscience, et que nous ne savons le plus souvent pas avec quelle ampleur et quelle profondeur la physis de l'homme corporel pénètre sa vie consciente ${ }^{25}$.

Le caractère originel de l'inconscience revêt donc une signification opposée à celle que peut lui accorder un disciple de Hegel : chez ce dernier, l'unité de la nature et de l'esprit réalisée par le Naturgeist dont traite l'anthropologie est comprise comme simple stade initial de l'esprit subjectif. L' « esprit naturel » ou «esprit-nature » n'est qu'une potentialité en attente de son actualisation à travers la conscience de soi, actualisation dont le lieu propre n'est pas la nature mais le domaine plus élevé de l'histoire. Il convient selon Löwith d'inverser les signes d'une telle téléologie et de " parcourir le chemin de Hegel et de Rosenkranz [...] également dans la direction opposée » afin de reconnaître que «le fondement durable de notre existence consciente est la vie inconsciente de la nature de toutes choses dont l'absence de savoir de soi n'équivaut en rien à une absence d'esprit $»^{26}$. Cette insistance, par exemple, sur la relation de dépendance qui subordonne l'activité de penser à l'irrigation du cerveau, n'est pas à comprendre, il va sans dire, dans le sens d'un réductionnisme naturaliste. S'il s'agit bien d'affirmer la compénétration intégrale de l'esprit et de la nature, et avec elle la thèse que tout esprit est « espritnature », sans doute l'essentiel est-il ici la prise en compte du problème schelerien de la "position » de l'homme dans le monde naturel : «Qu'est-ce que l'homme au sein de la totalité du monde ? » ${ }^{27}$ Comment caractériser un être appartenant entièrement à la nature et néanmoins capable de s'en distancer ? Où le situer, s'il n'est ni captif du monde ni extérieur à lui ${ }^{28}$ ? En cherchant à déterminer « la double nature de l'homme en tant qu'un être ni naturel ni spirituel, mais au contraire un être non-naturel », Löwith établit une fois pour toutes ce

\footnotetext{
${ }^{25}$ K. Löwith, Zur Frage einer philosophischen Anthropologie, SS 1, p. 340-341.

${ }^{26}$ Ibid., p. 341.

${ }^{27}$ K. Löwith, Max Scheler und das Problem einer philosophischen Anthropologie, SS 1, p. 220.

${ }^{28}$ Ibid., p. 241.
} 
qui constitue à ses yeux une donnée fondamentale de ce problème : le caractère premier d'une nature que l' «être vivant équivoque "hom$\mathrm{me}^{\prime \prime}$ » ne dépasse jamais que de façon relative ${ }^{29}$. Cette détermination, si elle traverse l'œuvre de Löwith, y reçoit néanmoins bientôt un sens et une portée tout à fait différents.

\section{Le tournant vers le cosmos}

Une question préalable devrait être ici posée, celle de la physionomie réelle de l'œuvre de Löwith, qui parait prendre un virage brutal vers le cosmos naturel, non sans lien avec des circonstances biographiques, en particulier le séjour de Löwith au Japon, de 1936 à $1940^{30}$. Certaines tensions présentes dans la thèse de 1928 peuvent en effet être lues comme autant de signes avant-coureurs d'un tel tournant: le parcours phénoménologique en quête de la Mitwelt paraît s'y déployer sur la toile de fond d'une question non tranchée, celle du statut à accorder à la nature, ce «fondement non-logique de $l^{\prime}$ existence ${ }^{31}$. Ainsi, il est permis de se demander si l'intention löwithienne de placer l'homme au centre de la considération n'a pas d'emblée pour condition de sa mise en œuvre un décentrement et un rejet de l'homme à la périphérie du véritable centre que serait dès lors la nature. Le tournant vers le cosmos apparaîtrait alors comme le prolongement et l'accomplissement du tournant vers l'anthropologie amorcé dans les années vingt par Löwith.

Comment cet approfondissement s'opère-t-il ? Par la prise en compte de la situation historique de l'homme. Dès son travail de 1935 sur Scheler, Löwith souligne ce qui constituera bientôt le motif de sa propre recherche: "ce qu'il y a d'authentique dans la personne et l'œuvre de Scheler, c'est [...] ce qu'il a saisi de la "perversion" fondamentale de tout notre monde humain moderne, monde qu'il a entendu restaurer en se remettant en quête d'ordres "éternels" et "naturels" propres à la vie »32. La question de la "position» de l'homme surgit dans le contexte historique d'une crise du rapport de l'homme à son monde, que Löwith n'interprète pas comme une

\footnotetext{
${ }^{29}$ K. Löwith, Das Individuum in der Rolle des Mitmenschen, SS 1, respectivement p. 33 et 34.

${ }^{30}$ Le tournant vers le cosmos a ainsi pu susciter la surprise de ses contemporains ; cf. le témoignage de Gadamer recueilli par J. Chitry, "Zur Wiedergewinnung des Kosmos. Karl Löwith contra Martin Heidegger ", in Zur philosophischen Aktualität Heideggers, vol. 2, D. Papenfuss et O. Pöggeler éd., Francfort-sur-le-Main, Klostermann, 1990, p. 98.

${ }^{31} \mathrm{~K}$. Löwith, Das Individuum in der Rolle des Mitmenschen, SS 1, p. 140.

${ }^{32}$ K. Löwith, Max Scheler und das Problem einer philosophischer Anthropologie, SS 1, p. 221.
} 
simple désorientation, mais comme la disparition de l'espace en lequel une orientation est en général possible. Dès lors, c'est la question du monde lui-même qui se pose: y a-t-il seulement une « nature » à laquelle il serait possible de se référer en déterminant la «nature » de l'homme? Ou au contraire faut-il établir le constat d'une absence de monde telle qu'une anthropologie ne serait plus envisageable que sous réserve d'un profond renouvellement et d'une réflexion sur le monde comme tel? Un tel élargissement de la problématique est indiqué, notamment, par la seconde préface à la thèse de 1928, qui souligne la nécessité d'une reprise, «dans un contexte plus large », de la «question globale du rapport de l'homme au monde, au sein duquel monde commun et monde alentour ne sont que des mondes relatifs $»^{33}$. Néanmoins, il ne s'agit pas seulement de reconnaître que ces mondes "relatifs » doivent être resitués au sein d'un monde naturel plus vaste: "monde» et "monde humain » apparaissent non seulement comme deux champs qui ne se recouvrent pas, mais comme deux instances qui s'opposent.

Cette opposition est un outil essentiel à la compréhension de la « perte de monde » (Weltverlust) ${ }^{34}$ qui constitue aux yeux de Löwith le ressort principal de la désorientation de l'homme moderne. De façon significative, c'est sous l'angle de la «critique de l'existence historique ", dont Histoire et Salut (1949) est le moment le plus spectaculaire, que Löwith aborde cette question. En effet, la perte du monde est principalement à rapporter, selon lui, à l'essor de la conscience historique, dans la mesure où celle-ci constitue l'histoire en tant qu'un monde à part entière qui vient supplanter le monde naturel. L' ' ' historisme » mis après-guerre sur la sellette par Löwith a le sens d'une " absolutisation ${ }^{35}$ de l'histoire, une universalisation du devenir par laquelle la nature se trouve relativisée ${ }^{36}$ - relativisée à un sujet qui, produisant ses propres déterminations, les déploie à titre de «monde ». L'historisme est un aspect - pour Löwith, l'aspect essentiel d'un subjectivisme, d'une «humanisation » massive par laquelle la nature comme telle se trouve annihilée. De cet historisme, Löwith établit une généalogie critique qui vise à mettre en évidence l'occultation du monde naturel et ce faisant ouvrir la possibilité de son dépassement. Cette généalogie restreinte de l'historisme s'inscrit dans une généalogie générale du nihilisme. Dans la première, Löwith fait

\footnotetext{
${ }^{33}$ K. Löwith, Das Individuum in der Rolle des Mitmenschen, SS 1, p. 14.

${ }^{34} \mathrm{~K}$. Löwith, Die Dynamik der Geschichte und der Historismus, in Weltgeschichte und Heilsgeschehen. Zur Kritik der Geschichtsphilosophie, Sämtliche Schriften Band 2, Stuttgart, J. B. Metzler, 1983, p. 306.

${ }^{35} \mathrm{~K}$. Löwith, Mensch und Geschichte, SS 2, p. 355.

${ }^{36}$ Cf. K. Löwith, Natur und Geschichte, SS 2, p. 284.
} 
remonter à Descartes la «théorie des deux mondes » opposant la nature à l'esprit ${ }^{37}$. La seconde reconduit, elle, jusqu'à la Bible, dans un mouvement analogue à celui qu'esquisse Histoire et Salut, en portant au jour les "présupposés théologiques de la philosophie de l'histoire ». Sans doute est-ce que le sens propre de la contribution de Löwith au débat sur la "sécularisation" s'y donne à voir. Cette généalogie est en effet une véritable histoire du monde, plus exactement une histoire de la disparition du monde, en laquelle le "monde humain ", sous la forme du "monde historique », vient mettre fin au monde comme tel. Le conflit interne à cette histoire du monde met ici aux prises "sphère de la physis » et « horizon de l'histoire », " nature de l'homme » et « existence historique ».

Le monde devient notre monde. Le cosmos physique supra-humain tombe dans l'oubli, et le monde se voit humanisé de fond en comble. Le monde devient monde humain. En même temps que le monde disparaît, la nature humaine s'évanouit pour laisser place à une existence historique ${ }^{38}$.

Aux yeux de Löwith, l'histoire du monde commence avec les Grecs, pour lesquels la nature subsiste par elle-même et repose sur elle-même. Le «monde », dans la pensée présocratique et même chez Platon, est un cosmos indépendant des choses humaines, qui doivent au contraire se régler sur cet ordre cosmique premier pour pouvoir être véritablement ordonnées. La doctrine biblique de la création vient s'opposer radicalement à une telle vue, en subordonnant le monde non seulement à un Créateur mais aussi à une créature faite à l'image de ce dernier.

Mais lorsque le cosmos physique n'est plus reconnu comme ce qu'il y a de plus éminent et de mieux ordonné, ou seulement comme ce qu'il y a de plus grand parmi les choses visibles, lorsque le plus haut et le meilleur, le summum bonum, est un Dieu créateur invisible mais digne de foi et que le monde tout entier est une création personnelle et cependant passagère, alors est inauguré, en passant par la voie du Dieu biblique, un concept anthropologique de monde qui est également le fondement de notre concept historique de monde. L'avènement du Salut devient histoire mondiale (Das Heilsgeschehen wird zur Weltgeschichte). De création $d u$ monde voulue par Dieu, le monde devient un monde pour l'homme ${ }^{39}$.

La Bible accomplit ainsi une négation du monde dont procède l'acosmisme moderne: avec l'idée chrétienne d'un monde créé à

\footnotetext{
${ }^{37}$ Cf. notamment K. Löwith, Geschichte und historisches Bewußtsein, SS 2, p. 421 sqq.

${ }^{38} \mathrm{~K}$. Löwith, Welt und Menschenwelt, SS 1, p. 302.

${ }^{39}$ Ibid., p. 304.
} 
dessein de l'homme, «le monde naturel est dépotentialisé et dénaturalisé (depotenziert und denaturiert) ; il lui manque 1'“à partir de soi" fondamental du mouvement et de la conservation propres qui sont ceux de la physis $»^{40}$. Il est alors domestiqué sous la forme de l'oikoumènè, du lieu de l'habitation humaine. Le "cosmos toujours persistant " n'est plus que "saeculum», "temps passager du monde $»^{41}$, soit de «ce » monde, «bas » monde voué à la ruine. Cette rupture décisive avec le «monde » grec est aux yeux de Löwith le grand événement qui projette son ombre sur la modernité, et qui donne son sens ultime à la sécularisation dont procède cette dernière. En effet, si la sécularisation est "sécularisation du saeculum chrétien ${ }^{42}$, elle redouble le processus de «dé-naturation » du cosmos en achevant son humanisation. Le saeculum chrétien, en tant que négation du cosmos subsistant par lui-même, est un "non-monde " qui, détaché de son substrat divin, est à son tour " mondanisé »: vidé de sa substance, il n'en tient pas moins lieu de monde à l'homme moderne. En d'autres termes, le «monde» propre à l'époque moderne est le résultat d'une "mondanisation du monde démondanisé ${ }^{43}$. Ce «pseudo-monde» n'est autre que l'histoire, qui n'est pas et ne peut pas être un monde au sens propre : ici, rien de stable, mais seulement un processus infini; rien d'existant par soi, mais seulement une production de l'homme par et pour lui-même; rien enfin qui y soit effectivement présent, puisqu'au contraire le sens de toute chose s'y trouve suspendu à l'avenir.

Tel est donc le paradoxe qui donne son impulsion et sa tonalité propres à la pensée anthropologique de Löwith: l'humanisation radicale du monde fait obstacle à une véritable pensée de l'homme. En effet, " pour pouvoir parler de la nature de l'homme, on doit au moins avoir une idée de la nature en général et comme telle $»^{44}$. Une connaissance de l'homme doit être une pensée du monde, et « dans la mesure où l'homme n'est pas une créature extra-mondaine, ni un être fait à l'image de Dieu, l'anthropologie philosophique a besoin du fondement d'une cosmologie ${ }^{45}$. Qu'est-ce en effet qu'une " anthropologie philosophique»? Dès lors que la philosophie comme telle vise le «tout», elle a ceci de propre qu'elle aborde «l'homme en tant

\footnotetext{
${ }^{40}$ Ibid., p. 305.

${ }^{41}$ Ibid., loc. cit.

${ }^{42}$ Ibid., p. 306.

${ }^{43}$ Loc. cit.

${ }^{44}$ K. Löwith, Natur und Humanität des Menschen, SS 1, p. 266.

${ }^{45}$ Loc. cit.
} 
que tel et en totalité ${ }^{46}$. Totalité qui revêt à son tour deux sens. Il s'agit en effet d'envisager non seulement l'homme comme un tout-comme « animal rationnel »- mais également au sein de la totalité à laquelle il appartient, comme "fragment » du tout ; et " c'est seulement avec la question englobante du rapport de l'homme au monde que l'anthropologie devient philosophique ${ }^{47}$. L'anthropologie à venir doit être une "anthropo-cosmo-logie » dont le centre de gravité est le cosmos lui-même, autrement dit une "considération excentrique ${ }^{48}$ dont le foyer est un "monde pré-humain et supra-humain [...] reposant entièrement sur lui-même et se conservant lui-même ${ }^{49}$. L'anthropologie est certes science de l'homme, mais la possibilité d'une telle connaissance repose sur le «logos de l'être humain » en tant que tel $^{50}$, qui renvoie à son tour à une instance plus fondamentale, le logos immanent au cosmos: la "cosmologie n'est pas une vue anthropologique du monde, bien au contraire le cosmos physique a lui-même un $\log o s »^{51}$. Si bien que le logos de l'anthropologie est moins l'appareil conceptuel construit par l'esprit humain pour se saisir réflexivement de lui-même qu'un ordre puisé au monde même en vue de concevoir le fragment humain de ce monde.

Penser l'homme comme partie du monde, inscrire l'anthropologie dans le cadre d'une cosmologie ne signifie pourtant pas renoncer à la différence anthropologique. Löwith maintient le principe de la conscience et souligne la capacité de l'homme à s'interroger sur lui-même dans un rapport distancié au monde: la "scission sujet-objet» est selon lui indépassable, car «constitutive pour l'être humain $»^{52}$. L'homme est un être questionnant, et à ce titre un " "être des lointains" qui transcende $»^{53}$. Toutefois, Heidegger comme Scheler ont commis l'erreur de comprendre cette transcendance comme un dépassement de soi vers son autre - l'être ou Dieu ${ }^{54}$, tandis que la différence anthropologique doit être conçue non par le détour d'une illusoire extériorité, mais dans son contexte propre, celui de la nature.

Si [...] l'on part de l'idée que l'homme n'est pas une âme sans corps, ni un esprit sans nature, ni non plus un Dasein purement existant, mais au

\footnotetext{
${ }^{46}$ K. Löwith, Zur Frage einer philosophischen Anthropologie, SS 1, p. 329.

${ }^{47}$ Ibid., p. 330.

${ }^{48} \mathrm{~K}$. Löwith, Welt und Menschenwelt, SS 1, p. 313.

${ }^{49}$ Ibid., p. 295.

${ }^{50}$ K. Löwith, Zur Frage einer philosophischen Anthropologie, SS 1, p. 329.

${ }^{51}$ K. Löwith, Welt und Menschenwelt, SS 1, p. 295.

${ }^{52}$ K. Löwith, Zur Frage einer philosophischen Anthropologie, SS 1, p. 333.

${ }^{53}$ K. Löwith, Natur und Humanität des Menschen, SS 1, p. 290.

${ }^{54}$ Cf. ibid., SS 1, p. 263.
} 
contraire une nature humaine - avec tous ses paradoxes et toutes ses ambigü̈tés - alors son transcender, s'il doit vraiment correspondre à sa nature, devrait être un transcender naturel. Le fait que l'homme interroge la nature en lui et autour de lui ne signifie pas qu'il devrait la dépasser vers une transcendance quelconque pour pouvoir vivre en tant qu'homme. Il se pourrait que le dépassement par lequel l'homme et son langage se différencient de l'animal s'accomplisse encore au sein de la sphère indépassable de la nature, sans s'étendre à un au-delà de l'étant par nature, si bien que le terminus a quo et le terminus ad quem $d u$ dépassement proprement humain serait un et le même : la nature ${ }^{55}$.

C'est à partir de cette thèse de l'immanence de l'homme au monde que l'ensemble des propositions de la période antérieure concernant la dualité ontologique de l'homme peut être repris sur un plan nouveau. Le monde en tant qu'ordre cosmique sera le fondement sur lequel ces propositions pourront s'appuyer. Si le monde est « l'unité et la totalité de tout ce qui est par nature », cette unité s'articule comme un «système » de déterminations réciproques cohérentes auquel l'homme appartient au même titre que tout être naturel, de sorte que son logos exprime celui du cosmos même ${ }^{56}$. Cet ordre est avant tout un rythme vital battu par les « rapports cosmiques » inconsciemment à l'œuvre dans la vie humaine, ces « rapports anonymes de notre vie corporelle à la vie planétaire, solaire, lunaire » qui déterminent les Stimmungen fondamentales de la vie ${ }^{57}$. Détaillant les déterminations naturelles internes de l'homme, Löwith ne craint pas de reprendre la doctrine antique des «tempéraments ». Il évoque également les « dispositions » naturelles, les talents et les dons, les inclinations, mais aussi les âges de la vie, et le caractère essentiel de la sexualité ; enfin, nous l'avons vu, est soulignée l'alternance fondamentale de la veille et du sommeil ${ }^{58}$. Toutes déterminations qui ne légitiment plus seulement l'anthropologie comme telle, mais plus résolument le «tournant vers la nature » qui la sous-tend ${ }^{59}$, et le caractère central du cosmos pour cette anthropologie.

Cependant, la question se pose de l'accès à la "nature », au fondement « cosmique » de l'anthropologie, si la modernité se caractérise justement par une éclipse totale du «monde naturel»: est-il seulement possible de déborder l'horizon historique et, dans l'hypothé-

\footnotetext{
${ }^{55}$ Ibid., p. 291.

${ }^{56}$ Cf. K. Löwith, Welt und Menschenwelt, SS 1, p. 296, et Die Natur des Menschen und die Welt der Natur, op. cit., p. 46 sq.

${ }^{57}$ Cf. K. Löwith, Zur Frage einer philosophischen Anthropologie, SS 1, p. 334.

${ }^{58}$ Cf. ibid., p. 335 sqq.

${ }^{59}$ Cf. O. Marquard, «Sur l'histoire du concept philosophique d" "anthropologie” depuis la fin du XVIII siècle », op. cit., p. 141 et 162 ; «Anthropologie», op. cit., col. 370.
} 
tique champ anhistorique ainsi ouvert, de circonscrire un authentique « concept naturel de monde »? Y a-t-il un "savoir» de la nature qui, au-delà de la « compréhension » historique, soit en mesure de dégager la «naturalité » même de la nature ${ }^{60}$ ? La difficulté surgit ici sur l'arrière-plan du paradoxe qui hante l'œuvre de Löwith en tant que projet de dépassement du paradigme historique par le moyen de sa critique historique: la référence constante à la physis grecque n'indique-t-elle pas en effet le recours à une interprétation de la nature tout aussi «historique» que sa représentation moderne? Löwith tente de lever cette difficulté en avançant deux arguments. D'abord celui d'une attestation "phénoménologique» de la nature: il y a incontestablement une expérience de la nature, hors de nous et en nous; le monde naturel est « là » et « cette nature apparaît en tout ce qui est, y compris le phénomène que nous nommons "homme" »61. De sorte $q u$ 'il serait permis de parler d'une expérience immédiate de la «nature humaine » elle-même, dont participerait la reconnaissance $\mathrm{d}^{\prime}$ autrui ${ }^{62}$. Il serait revenu - tel est le deuxième argument de Löwith à l'Antiquité grecque de faire une telle expérience de la nature et de l'articuler en une conceptualité frappée expressément pour elle. Le « retour impossible » à l'époque révolue des Grecs ne serait dès lors qu'un faux problème, n'ayant de sens que dans l'horizon étroit de "notre conscience historique ». Le recours à la physis est assumé par Löwith «avec la conviction que les Grecs ont fait une découverte qui - comme toute découverte initiale - demeure à jamais vraie ${ }^{63}$. Sa légitimité est en somme heuristique : dans la situation d'occultation du monde naturel qui est la nôtre, «il faut s'orienter grâce à une conception ancienne pour laquelle le monde était un cosmos physique $" 64$. Dès lors, la "compréhension » historique de la pensée grecque vise un point qui excède son propre horizon, et ce faisant échappe à tout relativisme « historiste »: l'expérience de la nature faite par les Grecs. Le cosmos grec, en articulant cette expérience fondatrice, procure le fil conducteur qui doit mener à l'air libre $\mathrm{l}^{\prime}$ « habitant de la caverne du monde historique » qu'est l'homme moderne, et le placer face à la " puissance élémentaire et à la grandeur monotone du monde, monde qui n'est pas nôtre, qui ne renvoie nullement à nous comme ce "à

\footnotetext{
${ }^{60}$ Cf. K. Löwith, Natur und Humanität des Menschen, SS 1, p. 268-269.

${ }^{61} \mathrm{Ibid} .$, p. 264.

${ }^{62}$ Sur ce « sentiment naturel » de l'humanité, cf. K. Löwith, Die Einheit und die Verschiedenheit des Menschen, SS 1, p. 256 sqq.

${ }^{63} \mathrm{~K}$. Löwith, Welt und Menschenwelt, SS 1, p. 314.

${ }^{64}$ Ibid., p. 298.
} 
dessein" de quoi il existerait, mais qui renvoie au contraire à luimême et à lui seul $»^{65}$.

L'expérience du monde nu, délivré de l'humanité, paraît constituer l'enjeu ultime du "point de vue anthropologique » de Löwith dans son ensemble. Un tel "point de vue » est loin de produire une anthropologie pleinement déployée à travers une détermination richement détaillée de la nature humaine. Si l'on met à part la thèse de 1928, et certains développements consacrés à la mort et au langage, le propos anthropologique de Löwith demeure assez succinct ; et il n'est pas illégitime de s'interroger sur la réalité de l'apport de Löwith à l'anthropologie du $X X^{e}$ siècle. Sans doute convient-il de chercher ailleurs l'essentiel du projet de Löwith, et de le concevoir moins comme une contribution à l'anthropologie que comme un plaidoyer pour l'anthropologie comme telle, c'est-à-dire plus profondément comme une recherche des conditions de possibilité de son instauration véritable. C'est ce à quoi s'emploie Löwith en réinscrivant l'interrogation anthropologique au cœur d'une ample réflexion historique mettant en évidence l'oubli moderne du cosmos. Le motif ne manque pas d'évoquer l'histoire de l'être heideggérienne, mais peut-être renvoie-t-il surtout à un penseur qui a su redécouvrir la «nature oubliée », et pointer "la vanité que possède le plus modeste à se sentir "l'homme" dans la nature et le monde ${ }^{66}$ : Nietzsche, dont Löwith situe "l'intention positive» dans la "reconquête du monde naturel» et dans la "réinsertion de l'homme dans la nature $»^{67}$. Selon Löwith, Nietzsche est « le premier et le seul à avoir mis en question l'idée traditionnelle de l'homme jusqu'à l'extrême limite sans pour autant la compromettre ${ }^{68}$. Il a tenté de fonder - ou de refonder : telle est la question qui surgit de l'interprétation de l'histoire au prisme de l'opposition de l'hellénisme et du christianisme menée aussi bien par Löwith que par Nietzsche - l'humanité de l'homme sur la nature, et de concevoir l'homme dans le contexte de la physis et ainsi en étroite proximité avec l'animal. Cette intuition nietzschéenne, remarque Löwith, a trouvé des développements chez Portmann, Plessner, Gehlen, mais le «monde vivant» et l' « homme corporel » ainsi redécouverts n'ont pas encore, estime-t-il, reçu suffisamment d'attention $^{69}$. À l'heure où, dans le monde francophone, on

\footnotetext{
${ }^{65}$ Ibid., p. 313.

${ }^{66}$ Cf. F. Nietzsche, Le voyageur et son ombre, $\S 304$ et 327.

${ }^{67} \mathrm{~K}$. Löwith, «Nietzsche et l'achèvement de l'athéisme », trad. M. de Gandillac, in Nietzsche aujourd'hui ?, vol. 2, Paris, Union Générale d'Éditions, 1973, p. 212 et 225.

${ }^{68} \mathrm{~K}$. Löwith, Natur und Humanität des Menschen, SS 1, p. 276.

${ }^{69}$ K. Löwith, Natur und Humanität des Menschen, SS 1, p. 279-280.
} 
redécouvre l'anthropologie philosophique allemande, nul doute qu'il serait préjudiciable de laisser en dehors de la perspective les travaux de Löwith, d'autant plus que cette anthropologie elle-même a su lui rendre un discret hommage ${ }^{70}$.

${ }^{70}$ Cf. H. Plessner, Die Stufen des Organischen und der Mensch. Einleitung in die philosophische Anthropologie, Gesammelte Schriften Band IV, Francfort-sur-le-Main, Suhrkamp, 2003, p. 20. 